\title{
Trabajadores y prácticas recreativas. Otra mirada al mundo del trabajo en América Latina (1930-1950)
}

\author{
Workers and recreational practices. Other Perspective for the Latin America \\ Another look about the world of work in Latin America (1930-1950)
}

Juan Carlos Yáñez Andrade*

\begin{abstract}
Resumen: El presente artículo busca identificar las principales líneas de investigación que han marcado los estudios sobre las prácticas recreativas -el tiempo libre, el ocio y el turismo- en el mundo laboral latinoamericano durante los años 1930 y 1950, complementando los enfoques nacionales con las perspectivas transnacionales. Estas prácticas recreativas se dieron en el marco de los cambios que supuso el desarrollo de la cultura de masas, el populismo y el proceso de industrialización que vivieron diversos países del continente. Este recuento historiográfico permite concluir la gran cantidad de publicaciones sobre las prácticas recreativas, aunque muchas de ellas enmarcadas en los enfoques sociales y culturales de la historia del movimiento obrero de vertiente más clásica.
\end{abstract}

Palabras clave: trabajadores, prácticas recreativas, tiempo libre, ocio, turismo

\begin{abstract}
This article identify the main lines of research o recreational practices -free time, leisure and tourism- in the Latin America labor world during the 1930s and 1950s, complementing national approaches with transnational perspectives. These recreational practices took place in the context of the changes that led to the development of mass culture, the populism and the industrialization process experienced by numerous countries of this continent. This historiographic recount allows to conclude the large number of publications on recreational practices, although many of them framed in the classic social and cultural approaches of the labor movement.
\end{abstract}

Keywords: workers, recreational practices, free time, leisure, tourism

Recibido: 1 agosto 2019 Aceptado: 20 octubre 2019

\footnotetext{
* Doctor en Historia. Académico de la Universidad de Valparaíso. Director Alterno del Centro de Investigación en Innovación, Desarrollo Económico y Políticas Sociales (CIDEP-UV). Correo electrónico: juancarlos.yanez@uv.cl. Se agradece de manera especial a los evaluadores que ayudaron a mejorar sustancialmente el presente artículo. El presente artículo forma parte del proyecto FONDECYT de Iniciación N¹1190167 titulado “Los trabajadores se toman un descanso. Las políticas de promoción del tiempo libre y el turismo social en Chile: 1927-1973".
} 


\section{Introducción}

Las prácticas asociadas al tiempo libre, el ocio y el turismo, que pueden ser sintetizadas en el concepto de prácticas recreativas, fueron ganando espacio a partir de la década de 1930 y permiten mirar desde otra perspectiva el mundo del trabajo. Tal como señala Robert Castel $^{1}$, que los debates sobre el tiempo libre, el ocio y las vacaciones pagadas se hayan dado entre la década de 1930 y 1950 responde al mejoramiento de las condiciones laborales y la construcción de un espacio luego de la jornada que se comenzó a representar como propio, separando, con ello, el espacio productivo del doméstico, y la condición laboral de la del vagabundo.

Los Estados, los empresarios y los trabajadores promovieron la cultura del tiempo libre, el ocio y la recreación en el marco de una sociedad de consumo que se expandía, ofreciendo nuevos pasatiempos asociados a la industria del ocio y que posibilitó con las prácticas recreativas otra forma de "regular las costumbres", como señala Silvia Ospital2. Por otra parte, al ser un aspecto fundamental para explicar las transformaciones que se generan en el periodo de entre guerras, las prácticas recreativas permiten comprender los procesos de democratización que vivieron los países europeos y los Estados Unidos en un primer momento, y algunos países latinoamericanos posteriormente, gracias a las mejoras en las condiciones de vida de los trabajadores industriales luego de la Gran Depresión ${ }^{3}$.

Las prácticas culturales desarrolladas al alero de las nuevas formas de producción industrial fueron abordadas por los pioneros estudios de la nueva historia social inglesa, en especial por Eric Hobsbawm y E.P Thompson4. El énfasis en la cultura y la noción de formación, como se sabe, supuso un fuerte cuestionamiento a las visiones mecanicistas y economicistas que suponía, para algunos, la tradición marxista Por su parte, los historiadores marxistas latinoamericanos reconocieron en las luchas por la jornada de ocho horas y el descanso dominical, la condición necesaria -aunque no suficiente- para el desarrollo sindical, ofreciendo nuevos espacios de recreación y de cultura para el desenvolvimiento de las actividades autónomas de los trabajadores. La historiografía social posterior, -la llamada nueva historia social- ha mostrado cierto interés por estudiar las prácticas sociales y culturales asociadas al tiempo libre y la industria del ocio, aunque como complemento de la acción política y, por lo tanto, muy vinculada a la acción de los partidos de raigambre obrera ${ }^{5}$. En este sentido se han descuidado, como tendremos la

\footnotetext{
1 Robert Castel, Las metamorfosis de la cuestión social. Una crónica del salariado, Buenos Aires, Paidós, 1997.

2 Silvia Ospital, “Turismo y territorio nacional en Argentina. Actores sociales y políticas públicas, 1920-1940”, Estudios Interdisciplinarios de América Latina y el Caribe, N², 2005, pp. 63-84.

3 Juan Carlos Torre y Elisa Pastoriza, "La democratización del bienestar". En: J. C. Torre, (dir.) Los años peronistas (1943-1955), Buenos Aires, Editorial Sudamericana, 2002, pp. 257-312.

${ }^{4}$ Eric Hobsbawm, El mundo del trabajo. Estudios históricos sobre la formación y evolución de la clase obrera, Madrid, Crítica, 1987; E. P. Thompson, La formación de la clase obrera en Inglaterra, Madrid, Capitán Swing, 2012.

5 Juan Suriano, Anarquistas. Cultura y política libertaria en Buenos Aires 1890-1910, Buenos Aires, Manantial, 2001; Mirta Lobato, La vida en las fábricas. Trabajo, protesta y política en una comunidad obrera, Berisso (1904-1970), Buenos Aires, Prometeo Libros/Entrepasados, 2001; Hernán Camarero, A la conquista de la clase obrera. Los comunistas y el mundo del trabajo en la Argentina, 1920-1935, Buenos Aires, Siglo XXI Editores, 2007; Sergio Grez, Los anarquistas y el movimiento obrero. La alborada de "la Idea" en Chile, 1893-1915, Santiago, Lom Ediciones, 2007.
} 
ocasión de presentarlo a lo largo de este artículo, otros actores relevantes para pensar las prácticas recreativas, como el Estado y el empresariado.

A partir de un examen general de las investigaciones que se han abocado a estudiar las prácticas recreativas, el balance historiográfico muestra cierto desinterés por parte de los historiadores por estudiar el tiempo libre y la industria del ocio, lo que se puede explicar por la visión que se ha construido de dichas actividades, las que han sido vinculadas a lo lúdico, un pasatiempo de poca densidad histórica, como señala Elisa Pastoriza $^{6}$. Por otra parte, la idea de que las prácticas recreativas no debieran ser un objeto de estudio propio, al ser dependientes de las modalidades de producción y de los niveles generales de vida7, hizo que la historia social haya tendido a estudiar lo que ocurría dentro de la fábrica o faena productiva, descuidando la construcción social y cultural fuera de los espacios de producción ${ }^{8}$.

El presente artículo busca identificar las principales líneas de investigación que han marcado los estudios sobre las prácticas recreativas -el tiempo libre, el ocio y el turismoen el mundo laboral latinoamericano entre los años 1930 y 1950, complementando los enfoques nacionales con las perspectivas transnacionales. El marco temporal se explica porque durante la década de 1930 se multiplicaron los debates sobre el trabajo y las prácticas recreativas en el contexto de los efectos de la Gran Depresión y los desafíos por ampliar los derechos laborales. Si bien es posible trazar una línea de continuidad histórica entre los debates por la "regeneración de la raza" y los discursos higienistas del siglo XIX, en su interés por intervenir el mundo popular y garantizar pasatiempos acordes con las pautas sociales normadas, a partir de la década de 1930 es posible observar en el contexto de la mayoría de los países latinoamericanos dos realidades completamente nuevas con respecto al siglo XIX:

a) La reducción progresiva de la jornada de trabajo a ocho horas diarias y la obtención del sábado inglés en muchos países de la región, hizo que aumentaran consecuentemente las preocupaciones y debates de higienistas, políticos y trabajadores por ocupar las horas disponibles luego de la jornada de trabajo. Por su parte, una vez obtenidas las vacaciones pagadas durante la década de 1930, se dieron las condiciones para pensar una industria del ocio y turismo.

b) El Estado se transforma en un actor protagónico en cuanto a proveer mejores condiciones sociales, haciendo que muchas actividades asociadas a la Iglesia Católica y fundaciones de caridad comenzaran a ser asumidas por ministerios como el del Trabajo o Bienestar Social, dando forma a una nueva burocracia laboral9.

6 Elisa Pastoriza, “Nuevos objetos de la Historia: los estudios turísticos en una perspectiva comparada”, Anuario IEHS, N²7, 2012, pp. 323-330.

7 Gareth Stedman Jones, “¿Expresión de clase o control social? Crítica de las últimas tendencias de la historia social del 'ocio'" en Lenguajes de clase. Estudios sobre la historia de la clase obrera inglesa (1832-1982), Madrid, Siglo XXI, 1989, p.83.

8 Juan Carlos Yáñez, “Trabajo y políticas culturales sobre el tiempo libre: Santiago de Chile, década de 1930”, Historia, N49, 2016, pp. 595-629.

${ }^{9}$ No podemos dejar de mencionar los propios cambios que experimenta el movimiento obrero hacia la década de 1930, tal como los describe el historiador uruguayo Rodolfo Porrini: “Este cambio estuvo ambientado por la 


\section{Las prácticas recreativas}

Conceptualmente la noción de prácticas recreativas busca entender fenómenos asociados al tiempo libre, el ocio e incluso el turismo, en un marco contextual mucho más amplio, las cuales son modeladas por marcos institucionales que dieron forma a una política pública sobre la materia. Esta mirada desde arriba (lo institucional) y desde abajo (las prácticas), obliga a trazar el surgimiento de los discursos en pro de la promoción de dichas prácticas, cómo se desarrollan en concreto y cómo se fundamentan como parte de una política social, así como analizar la consolidación, por una parte, de un aparato normativo e institucional (burocrático) y, por otra, de un mercado de bienes y servicios.

Conceptualmente la historiografía ha hecho una distinción entre tiempo libre, ocio y turismo, siguiendo el debate de la época, ya que el primero decía relación con las actividades promovidas por las agencias gubernamentales, el empresariado y los sindicatos para ocupar el tiempo disponible luego de la jornada de trabajo ${ }^{10}$. Por su parte la noción de ocio, que aparece en algunos documentos de la época, se entiende como complementario al tiempo libre, es decir como pasatiempo (descanso) o actividad de distracción (placer) ${ }^{11}$. En tanto que el turismo implica los esfuerzos de los mismos actores para promover actividades de desplazamiento de una ciudad o localidad a otra, la que involucra más de un día (vacaciones). En este sentido, el uso del concepto de prácticas recreativas tiene la ventaja de integrar estas diversas realidades (tiempo libre, ocio y vacaciones), junto con poner el foco de la investigación histórica en las experiencias vividas y compartidas, (a través del registro de folletos, relatos, postales, entre otros), y crecientemente formalizadas de recreación. De esta forma, se puede avanzar en superar ese paradigma que opone ocio y trabajo, apelando a que existe un abanico mucho más complejo de prácticas de esparcimiento entre ambos extremos.

Si bien las prácticas turísticas son de antigua data, las cuales se vinculan a espacios de esparcimiento propios de las elites ${ }^{12}$, el turismo social o de masas surge a partir de la década de 1930 en el contexto de una serie de procesos asociados a las ideologías nacionalistas, las luchas de los obreros por la reducción del tiempo de trabajo y la consolidación de una estructura y oferta turística cada vez más variada ${ }^{13}$. En este proceso

atenuación de las prácticas anti-sistema, la nacionalización y renovación de la clase obrera y nuevas camadas militantes en sus integraciones; también por las luchas comunes, antidictatorial en lo interno y antifascista a nivel mundial, y el efecto de la política de Frente Popular impulsado por la Internacional Comunista", Rodolfo Porrini, "Formas de la cultura alternativa: las 'veladas' y los festivales de las izquierdas uruguayas (Montevideo, 1920-1950)", Izquierdas, N49, 2020, pp. 222-242.

${ }^{10}$ Los trabajos sobre el paternalismo industrial y las actividades recreativas propuestas a los trabajadores han tenido un importante desarrollo en los últimos años. Véase, por ejemplo, el número especial de la revista Avances del Cesor sobre el paternalismo industrial en América Latina, en particular la introducción al dossier de Silvia Simonassi y Laura Badaloni, "Trabajadores, empresas y comunidades urbanas: reflexiones introductorias", Avances del Cesor n¹0, 2013, pp. 101-111. Para el caso de Chile, véase Enzo Videla, Hernán Venegas y Milton Godoy (edit.), El orden fabril. Paternalismo industrial en la minería chilena. 1900-1950, Valparaíso, Editorial América en movimiento, 2016.

11 Norbert Elias y Eric Dunning, Deporte y ocio en el proceso de la civilización, México, FCE, 1992.

12Alain Corbin, El territorio del vacío. Occidente y la invención de la playa, Barcelona, Mondadori Grijalbo, 1993.

13 Bertram Gordon, "El turismo de masas: un concepto problemático en la historia del siglo XX", Historia Contemporánea, N²5, 2002, pp. 125-156. 
de generación de lo que se ha dado en llamar una industria del ocio, tuvo un papel importante el Estado que vio en las actividades recreativas y de esparcimiento una válvula de escape para las tensiones propias de la vida urbana, así como una instancia de integración social y de expansión del consumo según fuese el caso de las ideologías imperantes.

Los Estados, de modo general, ayudaron a la promoción del turismo social en al menos tres aspectos diferentes, con distintos grados de desarrollo, aunque complementarios. Primero, hubo una política transversal a nivel latinoamericano en incorporar reformas a los sistemas laborales, garantizando una jornada laboral de ocho horas y semanal de 48 a partir de las décadas de 1920 y 1930. Se desarrollaron instancias de promoción y de información turística a través de los Departamentos de Turismo u Oficinas de Información Turística que se crearon en América Latina, permitiendo que sectores de la clase obrera accedieran a balnearios y centros vacacionales de manera más regular ${ }^{14}$. En segundo lugar, el Estado ejecutó obras viales, creó los primeros parques nacionales y mejoró reglamentaciones que hicieron rutinarias las actividades de esparcimiento. Por ejemplo, los balnearios costeros, por su relativa cercanía con las capitales sudamericanas, fueron espacios privilegiados para promover los primeros paseos populares, aunque en los países con amplios territorios, muchos de los cuales eran inhóspitos, el turismo se transformó en una instancia de "colonización interna" que permitió unificar el país y ofrecer nuevas postales al imaginario nacional ${ }^{15}$. En tercer lugar, en algunos países el Estado creó de manera pionera los primeros centros de formación y educación en materia turística, como una forma de profesionalizar la industria y ofrecer un campo laboral nuevo. Chile presenta una de las experiencias inéditas con el Instituto de Educación Hotelera creado por el Ministerio de Fomento en 1929 bajo la presidencia de Carlos Ibáñez del Campo, institución que nos permite conocer las transformaciones y exigencias nuevas que se producen a nivel del Estado a partir de la formulación de las primeras políticas públicas en materia turística ${ }^{16}$.

Una de las primeras deficiencias que pueden mostrar los estudios que se han hecho sobre el tiempo libre y el turismo social en América Latina -aunque reconociendo su importancia- es que adolecen de una visión integral y de largo plazo, conociendo muy pocas historias sobre el tiempo libre, el ocio o el turismo a gran escala, destacando como una excepción el libro de Fernando Armas para el caso del Perú en el siglo XIX y XX, y el de Rodolfo Porrini para el caso de Montevideo (Uruguay) entre 1930 y 195017. En un diagnóstico un tanto diferente Ospital ${ }^{18}$ señala que, si bien el tiempo libre y el turismo son

\footnotetext{
14 José Faraldo y Carolina Rodríguez-López, Introducción a la historia del turismo, Madrid, Alianza Editorial, 2013.

15 G. Silvestre, "Postales argentinas". En: C. Altamirano (Ed.) La Argentina en el siglo XX, Buenos Aires, Ariel, 1999, pp. 111-135; L. Vejsbjerg, P. Núñez, y B. Matossian, “Transformation of Frontier National Parks into tourism sites. The North Andean Patagonia Experience (1934-1955)", Alma Tourism, N¹0, 2014, pp. 1-22.

16 Juan Carlos Yáñez, “El Instituto de Educación Hotelera de Chile: Una experiencia pionera de formación en el campo de la hospitalidad", Estudios y Perspectivas en Turismo, Nº1, 2017, pp. 178-193.

17 Fernando Armas, Una historia del turismo en el Perú. El Estado, los visitantes y los empresarios (1800-2000), Lima, USMP, 2018, vol. 1-2; Rodolfo Porrini, Montevideo, ciudad obrera. El tiempo libre desde las izquierdas (1920-1950), Montevideo, Universidad de la República, 2019.

18 Silvia Ospital, op. cit.
} 
un objeto de estudio historiográfico reciente, los historiadores se han interesado en investigar este campo por la multiplicidad de enfoques que permite, sintiéndose muy cómodos al analizar sus dimensiones políticas, sociales, culturales o económicas. Es probable que esta multiplicidad de abordajes y la multidimensión que adquieren las prácticas recreativas, expliquen, en parte, la fragmentación que se observa en los estudios que abordan la temática.

\section{Enfoques historiográficos}

Los abordajes sobre el origen y desarrollo del tiempo libre, el ocio y el turismo pueden ser organizados desde distintos enfoques historiográficos: un enfoque sociocultural, político y económico, aunque no siempre es posible establecer una delimitación clara entre ellos.

\section{Enfoque sociocultural}

En su dimensión sociocultural las prácticas recreativas se entienden como un proceso de larga duración que se prolonga hasta el día de hoy gracias a la globalización, donde cada vez una mayor cantidad de personas pueden utilizar el tiempo libre y las vacaciones como vías para acceder a los bienes culturales de otras regiones (del propio país o del mundo), anteriormente reservados a exploradores, peregrinos o las elites ${ }^{19}$. Los estudios sobre el tiempo libre y el ocio permiten un acercamiento a las prácticas fuera de la jornada de trabajo, de naturaleza emancipadora y que va más allá de las necesidades básicas, instancias promovidas tanto por las autoridades preocupadas por ofrecer pasatiempos acordes a la moral, como por la dirigencia obrera interesada en promover escuelas de formación sindical ${ }^{20}$.

Lo interesante de los estudios sobre el ocio y el tiempo libre es que han permitido renovar la noción de sociabilidad, concepto que adquirió relevancia a partir de la Escuela de los Anales y que busca integrar en un análisis comprensivo las diferentes formas de participación colectiva (clubes, cafés, organizaciones políticas, agrupaciones de inmigrantes, entre otros) y prácticas culturales (lecturas, rituales, festividades, entre otros) de grupos determinados. En este sentido hay dos tipos de estudios que han integrado las prácticas recreativas dentro de un enfoque sociocultural:

a) En primer lugar, aquellos que indagan en el aporte de las prácticas recreativas a la formación de la cultura obrera. Los estudios sobre el anarquismo han destacado cómo los ácratas lucharon por construir un modelo educativo autónomo a la cultura dominante y

19 M. Smith, Issues in cultural tourism studies, Nueva York, Routledge, 2003.

20 Mauricio Archila, "El uso del tiempo libre de los obreros. 1910-1945”, Anuario Colombiano de Historia Social y de la Cultura, N¹8-19, 1991, pp. 145-184; Yanina Leonardi, “Ocio y arte para los obreros durante el primer peronismo (1946-1955)", Revista Mundos do Trabalho, N¹2, 2014, pp. 239-249. 
formas de vida sanas al aire libre 21 , junto al rol de la literatura y la dramaturgia en la promoción de los valores libertarios ${ }^{22}$. En una vertiente vinculada a las prácticas de izquierda, el historiador uruguayo Rodolfo Porrini ha investigado las excursiones y los pic-nics como parte de las actividades al aire libre, alejadas de los salones y formas de manifestación cultural más tradicional ${ }^{23}$. En especial destaca las actividades destinadas al conjunto de la familia, aunque reconoce los problemas prácticos en poder integrar a las mujeres y esposas en estas actividades, al ser responsables del cuidado de los menores ${ }^{24}$. También dichos trabajos abordan los cuestionamientos a los pasatiempos más tradicionales del mundo popular uruguayo, como eran los carnavales. Por su parte el historiador argentino Hernán Camarero ha estudiado las prácticas emancipadoras al alero de los sindicatos y partidos obreros de izquierda, en especial el Partidos Socialista y el Partido Comunista de la Argentina ${ }^{25}$. En un trabajo sobre las prácticas de entretenimiento destaca las actividades promovidas especialmente para los niños, con el objetivo de alejarlos del mundo del trabajo y el abandono ${ }^{26}$. Además, de las actividades tradicionales de distracción de carácter más intelectual, como los ateneos, salones de lectura, bibliotecas, entre otros, se destacan para los niños las salidas campestres, las actividades deportivas y el cine. En el caso de los comunistas argentinos, si bien dichas prácticas apuntaban a la distracción, no perdían de vista la formación de una cultura militante de izquierda en los menores. Sin embargo, tanto en los enfoques que buscan rescatar las prácticas recreativas como elementos esenciales en la formación de la cultura de izquierda, así como en los enfoques que ven en dichas prácticas otra forma de militantismo, queda abierta la discusión de si las prácticas recreativas ayudaron o no a dar forma a una cultura obrera en pugna con la cultura dominante.

b) En segundo lugar, se puede nombrar a aquellos historiadores interesados en estudiar las prácticas recreativas desde un enfoque más vinculado a las representaciones. Siguiendo las perspectivas de la historia cultural, estos investigadores apuntan a reconocer en el Estado un "agente nacionalizador" que ayudó a la construcción de la identidad nacional y la participación de los sectores populares que anteriormente habían sido excluidos de la nación. Bárbara Silva y Rodrigo Henríquez, por ejemplo, han estudiado para el caso

\footnotetext{
21 Eduardo Godoy, "El discurso moral de los anarquistas chilenos en torno al alcohol a comienzos del siglo XX". En: Juan Carlos Yáñez (Editor), Alcohol y trabajo. El alcohol y la formación de las identidades laborales en Chile. Siglo XIX y XX, Osorno, Universidad de Los Lagos, 2008, pp. 121-144.

22 Pedro Bravo Elizondo, Cultura y teatro obreros en Chile, 1900-1930, Madrid, Ediciones Michay, 1986; Sergio Pereira, Antología crítica de la dramaturgia anarquista en Chile (Santiago de Chile: Editorial de la Universidad de Santiago, 2005); Sergio Grez, “ ¿Teatro ácrata o teatro obrero? Chile, 1895-1927”, Estudios Avanzados, N¹5, junio 2011, pp. 9-29.

23 Rodolfo Porrini, “Izquierda uruguaya y culturas obreras. Propuestas al 'aire libre': pic-nics y paseos campestres en Montevideo: 1920-1950", Revista Mundos do Trabalho 3(6), 2011, pp. 105-129.

24 Rodolfo Porrini, "Formas de la cultura alternativa: las 'veladas' y los festivales de las izquierdas uruguayas (Montevideo, 1920-1950)", op.cit.

${ }^{25}$ Hernán Camarero, A la conquista de la clase obrera, op. cit.

26 Hernán Camarero, "Experiencias de cultura obrera: las prácticas de entretenimiento y erudición de los socialistas y los comunistas durante los años 1920-1930", X Jornadas Interescuelas/Departamentos de Historia. Escuela de Historia de la Facultad de Humanidades y Artes, Universidad Nacional del Rosario. Departamento de Historia de la Facultad de Ciencias de la Educación, Universidad Nacional del Litoral, Rosario, 2005.
} 
chileno cómo el tiempo libre y las vacaciones ayudaron a configurar un nuevo sujeto social -como es el ciudadano nacional- el cual se reapropia de los espacios y paisajes nacionales y los resignifica en un contexto de creciente democratización ${ }^{27}$. Los sectores populares comienzan a participar de actividades que convocan a la unidad nacional, como los deportes, paseos al aire libre, viajes campestres o de playa, en una construcción identitaria que tiene como exigencia fundamental el garantizar las reformas sociales sin provocar temores en los sectores de la elite. El que muchas de las políticas de promoción del tiempo libre y de las vacaciones se hayan dado bajo el alero del Frente Popular en Chile (19381941) vendría a reforzar la idea de que las prácticas recreativas fueron la vía de integración de los trabajadores a la nación.

\section{Enfoque político}

En cuanto a la dimensión política, las prácticas recreativas han sido estudiadas en relación directa con la emergencia de las ideologías nacionalistas y populistas que asumieron el poder en Europa y América Latina durante los años 1930 y 1940, viendo en el turismo y el tiempo libre una nueva forma de integrar a la nación y vincular a los sectores de trabajadores con ciertos ideales nacionales ${ }^{28}$. A través de unos ejemplos representativos se puede establecer un panorama básico sobre el uso que los gobiernos le dieron al turismo como elemento de movilización político-social ${ }^{29}$. Es decidor que el uso que los investigadores le han dado al concepto de turismo social o de masas se vincule con la consolidación de los procesos de industrialización y de producción estandarizada a partir de los años 194030, lo que ha llevado a concebir la promoción del tiempo libre, el ocio y el turismo como una nueva forma de disciplinamiento de los trabajadores. Sin estar en contra de esta idea, consideramos más sugerente la reflexión de Silvia Ospital cuando señala que el Estado al promover una política turística puede ser "un regulador de las costumbres", lo que supone considerar que las prácticas recreativas también implicaron en los trabajadores experiencias de distracción, placer y emancipación ${ }^{31}$.

Reiterando lo que señala Bertram Gordon32, el turismo de masas "es un concepto problemático", porque de tanto usarlo para describir una realidad donde cada vez mayor cantidad de personas comenzaron a viajar a partir de los años 1930, terminó siendo una expresión peyorativa. En todo caso es correcto señalar que las ideologías nacionalistas

27 Bárbara Silva, "La espacialidad y el paisaje en las representaciones nacionales durante el Frente Popular chileno, 1938-1941", Revista de Historia Social y de las Mentalidades, N¹, 2018, pp. 129-153; Bárbara Silva y Rodrigo Henríquez, "El pueblo del Frente. Representaciones sobre la ciudadanía en Chile: 1930-1950", ERLACS, N¹03, 2017, pp. 91-108.

28 M. Schaffer, See America first: Tourism and national identity 1880-1940, Washington DC: Smithsonian Institution Press, 2001; E. Zuelow, A history of modern tourism, New York, Palgrave, 2016.

29 E. Tizzoni, “Les politiques touristiques du fascisme et les relations internationales de l'Italie, entre diplomatie publique et création d'une marque de destination-Italie”, Cahiers de la Méditerranée, N88, 2014, pp. 1-15

30 José Faraldo y Carolina Rodríguez-López, Introducción a la historia del turismo, Madrid, Alianza Editorial, 2013.

31 Silvia Ospital, op. cit.

32 Bertram Gordon, op. cit. 
utilizaron el turismo de masas como una forma de movilización y control de los trabajadores. Victoria de Grazia ${ }^{33}$ llevó a cabo un estudio pionero sobre la obra de organización del ocio y tiempo libre durante el fascismo italiano, destacando la labor de la Opera Nationale Dopo lavoro como esfuerzo para el control de los sindicatos. Para el caso de la Alemania nazi Shelley Baranowski ${ }^{34}$ analizó las políticas de turismo de masas y la acción de la Sociedad Nacional Socialista y el Frente Alemán del Trabajo en la promoción de viajes y las actividades recreativas, como el deporte y las excursiones.

En América Latina, Guillermo Cicalese ${ }^{35}$ aborda el concepto de turismo social para entender tanto las acciones del peronismo argentino en materia de recreación, como la acción de las corrientes socialistas. Sin embargo, este concepto había sido utilizado mucho antes por Eugenia Scarzanella ${ }^{36}$ para hacer referencia a los programas vacacionales durante el peronismo y sus ideales gubernamentales de valoración de lo nacional. El turismo social volvió a ser abordado por Elisa Pastoriza ${ }^{37}$ para el mismo periodo, dedicándose a describir las políticas públicas en materia de recreación y los rituales obreros en torno al ocio y el tiempo libre entre 1943 y 1955. Utilizando un enfoque comparado, Guillermo Comparato ${ }^{38}$ estudió la acción del gobierno de Vargas en Brasil y de Perón en Argentina, destacando el turismo como una categoría válida para repensar la noción de populismo. Lo mismo hace Cesar Capanegra, al estudiar la política turística argentina en el siglo $\mathrm{XX}^{39}$.

En un registro complementario, aunque no directamente vinculado con el populismo nacionalista, John Urry ${ }^{40}$ estudió el turismo de masas en Brasil durante los años 1930 y 1940 y el imaginario construido en torno a los balnearios. La formación de balnearios en las ciudades de Viña del Mar, Mar del Plata y Montevideo en perspectiva comparada ha sido también tratada en una obra colectiva dirigida por Elisa Pastoriza ${ }^{41}$, poniendo énfasis en las transformaciones urbanas y las negociaciones políticas entre distintos actores por la apropiación de los espacios de consumo y de recreación. Los parques nacionales han sido estudiados para comprender la ocupación del territorio como preocupación geopolítica y como instancia de valoración de lo nacional por parte de los gobiernos populistas, en

33 Victoria de Grazia, The Culture of Consent: Mass Organization of Leisure in Fascist Italy. New York, Cambridge University Press, 1981.

34 Shelley Baranowski, Strength Through Joy: Consumerism and Mass Tourism in the Third Reich, Cambridge: Cambridge University Press, 2004.

35 G. Cicalese, "Conflictos políticos, enredos jurídicos y negocios de verano en torno de las playas marplatenses". En: Elisa Pastoriza, Las puertas al mar. Consumo, ocio y política en Mar del Plata, Montevideo y Viña del Mar, Buenos Aires, Editorial Biblos, 2002, pp. 133-162.

36 E. Scarzanella, "El ocio peronista: vacaciones y "turismo popular" en Argentina (1943-1955)". Entrepasados N¹4, 1988, pp. 65-84.

37 Elisa Pastoriza, "El turismo social en la Argentina durante el primer peronismo. Mar del Plata, la conquista de las vacaciones y los nuevos rituales obreros, 1943-1955". Revista Nuevo Mundo, Débats, publicada el 16 de junio de 2008. URL: http:/ / nuevomundo.revues.org/36472

38 Guillermo Comparato, "Matices populistas: La política turística de Getulio Vargas (1937-1954) y de Juan Domingo Perón (1946-1952)”, Trashumante - Revista Americana de Historial Social N³, 2014, pp. 16-133.

${ }^{39}$ César Capanegra, “La política turística en la Argentina en el siglo XX”. Aportes y transferencias, N¹, 2006, pp. 43-61.

40 J. Urry, O olhar do turista: lazer e viagens nas sociedades contemporâneas, São Paulo: SESC/ Nobel, 1996.

${ }^{41}$ Elisa Pastoriza, Las puertas al mar. Consumo, ocio y política en Mar del Plata, Montevideo y Viña del Mar, Buenos Aires, Editorial Biblos, 2002. 
especial en Argentina42.

Como hemos visto, el papel del Estado resultó omnipresente en la promoción del turismo entre los trabajadores, especialmente por las exigencias normativas, de recursos y comunicacionales que había que movilizar, lo que ha hecho que la historiografía descuide el papel de otras instancias administrativas en el desarrollo del turismo. En Inglaterra, tanto Walton y Walvin han destacado el aporte de los municipios en la labor de recreación de los trabajadores, en especial en la zona de balnearios ${ }^{43}$. En uno de los pocos trabajos para América del Sur, Nelly Da Cunha estudió el papel del municipio de Montevideo en la promoción turística ${ }^{44}$.

\section{Enfoque económico}

En cuanto a la tercera dimensión, es decir la económica, los investigadores han destacado la importancia que tuvo el tiempo libre y el turismo social como factor dinamizador de la economía en un contexto de lenta recuperación luego de la Gran Depresión ${ }^{45}$. En América Latina el modelo de desarrollo económico que muchos países adoptaron a partir de la crisis de 1929 fue funcional a la promoción de un mercado interno y al aumento de las competencias interventoras del Estado, acompañado por la expansión de la educación pública, el sistema sanitario y un conjunto de políticas sociales, tal como lo estudian Lvovich y Suriano ${ }^{46}$ y Ramacciotti ${ }^{47}$ y Milanesio ${ }^{48}$ para el caso argentino. Para la misma Argentina, Silvana Osow ${ }^{49}$ investigó el turismo como parte de los planes quinquenales del peronismo (en especial el segundo de 1951) y una forma protagónica de entender el Estado en la economía, a través de la creación de zonas y centros turísticos, la promoción del turismo social (trabajadores), el escolar y el internacional. Para el caso de Chile el historiador Stephan Rinke estudió la formación de una lenta pero creciente sociedad de consumo bajo el influjo siempre ambiguo y conflictivo de los Estados Unidos, destacando en uno de sus capítulos la llegada de numerosos turistas norteamericanos ${ }^{50}$.

\footnotetext{
42 Melina Piglia, "En torno a los Parques Nacionales: primeras experiencias de una política turística nacional centralizada en la Argentina (1934-1950)", Pasos, N¹, 2012, pp. 61-73; Paula Núñez, "Marcas y atractivos en una provincia imaginada. Río Negro, Argentina, 1958-1976", Quinto Sol, N¹, 2018, pp. 1-24.

43 John Walton y James Walvin (edit.), Leisure in Britain, 1788-1939, Manchester, Manchester University Press, Oxford Road, 1983; John Walton, The British Seaside: holidays and resorts in the twentieth century, Manchester, Manchester University Press, 2000.

44 Nelly Da Cunha, Montevideo ciudad balnearia (1900-1950). El municipio y el fomento del turismo, Montevideo, Facultad de Humanidades y Ciencias de la Educación, 2010.

45 Paulo Drinot y Alan Knight, La Gran Depresión en América Latina, México, FCE, 2015.

46 D. Lvovich, y J. Suriano, Las políticas sociales en perspectiva histórica. Argentina, 1870-1952, Buenos Aires, Prometeo, 2006.

47 Karina Ramacciotti, La política sanitaria del peronismo, Buenos Aires, Editorial Biblos, 2009.

48 Natalia Milanesio, Cuando los trabajadores salieron de compras. Nuevos consumidores, publicidad y cambio cultural durante el primer peronismo, Buenos Aires, Siglo XXI Editores, 2014.

49 Silvina Osow, El desarrollo turístico en la Argentina durante el siglo XX: La política turística peronista (1946-1955), Tesis para obtener el grado de Licenciada en Sociología, Universidad Nacional de La Plata, 2010.

50 Stephan Rinke, Encuentros con el yanqui: Norteamericanización y cambio sociocultural en Chile (1898-1990), Santiago, Dibam, 2014.
} 
En este contexto, las políticas redistributivas y el aumento de las capacidades de consumo de los trabajadores fueron fundamentales en el proceso de recuperación económica, discutiéndose en distintos países el ingreso mínimo y las vacaciones pagadas de una o dos semanas ${ }^{51}$. El acceso a los medios de transporte y el mejoramiento de las vías de comunicación fueron aspectos importantes para sentir una cercanía a los espacios, incluidos los más lejanos ${ }^{52}$. Los clubes de automóviles fueron importantes promotores del turismo, supliendo en un comienzo la débil participación del Estado ${ }^{53}$. Por su parte, la temprana organización sindical de los trabajadores y empleados del turismo da cuenta de la importancia que comienzan a tener estas actividades en la economía, junto con comenzar a ser registradas en los censos.

Prueba de la consolidación del tiempo libre como factor asociado al compromiso con la productividad es el caso de Chile, cuyo Código Laboral de 1931 en su artículo 98 estableció que solo tendrían derecho a vacaciones de dos semanas con sueldo íntegro los operarios que hubiesen trabajado al menos doscientos ochenta y ocho días al año y de solo una semana aquellos que lo hubiesen hecho entre doscientos veinte y doscientos ochenta y siete $^{54}$. En este marco, las perspectivas higienistas concibieron el tiempo libre como una instancia para afrontar los problemas de fatiga y de accidentabilidad laboral. Es así como los espacios turísticos de la playa, el campo o las montañas comenzaron a ser relacionados con determinadas acciones curativas y terapéuticas, lo mismo que el alpinismo y excursionismo 55 .

En una variante de las anteriores perspectivas, Juan Carlos Yáñez estudió el discurso de fomento del turismo como actividad económica para los tempranos años de la segunda mitad de la década de 1920, bajo la presidencia de Carlos Ibáñez del Campo (1927-1931). Durante este periodo junto con promover el turismo como una actividad económica importante, el Estado creó un instituto pionero para la formación de personal especialidad en la hospitalidad, denomina Instituto de Educación Hotelera ${ }^{56}$

\section{Las perspectivas transnacionales}

Las perspectivas transnacionales ofrecen miradas interesantes para abordar estos procesos, porque muchos de los debates sobre el tiempo libre, la reducción de la jornada laboral, el esparcimiento y el consumo, se dieron en instancias internacionales difundidas

\footnotetext{
51 Karin Rosemblatt, "Masculinidad y trabajo: el salario familiar y el estado de compromiso, 1930-1950". Proposiciones N²6, 1995, pp. 70-86

52 G. Giucci, La vida cultural del automóvil. Rutas de la modernidad cinética, Buenos Aires, Universidad Nacional de Quilmes-Prometeo, 2007; Rodrigo Booth, “Turismo, panamericanismo e ingeniería civil: la construcción del camino escénico entre Viña del Mar y Concón (1917-1931)", Historia, N²47, 2014. pp.277-311.

53 Melina Piglia, "La incidencia del Touring Club y del ACA en la construcción del turismo como cuestión pública", Estudios y perspectiva en turismo, vol 17, 2008, pp. 51-70.

54 Juan Carlos Yáñez, "El control del tiempo de trabajo. El tiempo de trabajo. El establecimiento de la jornada laboral continua en Chile en 1942", Revista de Derecho, XLV, 2015, pp. 403-422.

55 Jorge Pedro Sgrazzutti, "La organización del "Tiempo Libre" en dictaduras europeas y en Argentina entre 1922 y 1955", Revista Historia Moderna i Contemporània, N², 2004, pp. 17-61.

56 Juan Carlos Yáñez, “El Instituto de Educación Hotelera”, op. cit.
} 
por intelectuales comprometidos en estos temas ${ }^{57}$. Por ejemplo, las discusiones sobre la reducción del tiempo de trabajo se apoyaron en las legislaciones de otros países y en la difusión de principios emanados de organismos internacionales. La jornada de ocho horas se extendió por el Cono Sur de América durante los años 1920 porque se adaptaba bien a la división tripartita del día, en consideración de que las personas podían complementarla con ocho horas de recreación y ocho horas de descanso, pero además porque se adaptaba a múltiples realidades, lo que explica la ola de reformas que se impuso en muchos países de manera simultánea.

Parece claro que los teóricos y legisladores que discutieron sobre las prácticas recreativas se apoyaron en los diagnósticos e ideas que circularon sobre el tema en distintos congresos y revistas especializadas. En este aspecto dos instancias jugaron un rol mayor en el reconocimiento de la importancia del tiempo libre como una política de Estado: los congresos internacionales y la OIT. En 1930 se reunió por primera vez el Congreso Internacional de Recreos en la ciudad de Lieja (Bélgica), que manifestó su deseo de que se constituyera una comisión internacional encargada de fomentar y coordinar los esfuerzos emprendidos por los países en materia de recreación ${ }^{58}$. Desde la década de 1930 se hicieron regulares los congresos sobre la organización de las horas libres y el recreo, aprovechando el alcance mundial de los Juegos Olímpicos. El primer encuentro se desarrolló en Los Ángeles, Estados Unidos, en 1932 y el segundo en la ciudad de Hamburgo en ocasión de las Olimpiadas de Berlín de 193659. El tercer congreso se desarrolló en Roma en 1938 y habría sido el primero en el cual participó un expositor chileno: el doctor José Gálvez ${ }^{60}$.

Los congresos panamericanos, por su parte, resolvieron en numerosas oportunidades ofrecer facilidades para que los turistas y los distintos medios de transporte, en especial los vehículos, pudieran disponer de mejores condiciones para traspasar las fronteras. En este sentido, la creación de una carretera y ferrocarril panamericano se entienden en esa dirección. De hecho, en la VII Conferencia Panamericana de 1933, realizada en Montevideo, se resolvió aprobar una recomendación sobre el fomento del turismo, proponiendo la reducción de trabas en la obtención de pasaportes y visados, y el reconocimiento de la categoría de turista a través de un pasaporte gratuito por tres meses, entre otras medidas.

Otra instancia internacional que ayudó a hacer visible el tema fue la OIT. En 1923 su Consejo de Administración decidió inscribir en la orden del día de la próxima conferencia la utilización del tiempo libre, para lo cual se inició una serie de encuestas entre los países miembros para conocer su situación y opinión al respecto. La conferencia internacional de 1924 aprobó una recomendación donde se sugirió la necesidad de que los gobiernos adoptaran medidas como el mejoramiento de los espacios de producción, el uniformar los beneficios en materia de tiempo libre y reducir al mínimo el traslado desde los domicilios

\footnotetext{
57 M. Linden, Historia transnacional del trabajo, Valencia, ned-Instituto de Historia Social, 2006.

58 OIT, Utilización del tiempo libre de los trabajadores. Informe presentado al Comité de Correspondencia de Recreos, Ginebra, OIT, 1952, p. 10.

59 Congreso Mundial, Memoria del Congreso Mundial para la organización de las horas libres y del recreo, Berlín, Verlag Freude und Arbeit, 1937.

${ }^{60}$ El Diario Ilustrado, Santiago, 21 de octubre de 1938.
} 
a los lugares de trabajo, junto con enumerar modalidades de utilización de las horas disponibles: la formación cultural y la creación de jardines obreros y campos deportivos ${ }^{61}$.

En 1936 la OIT dio un paso importante al reconocer el derecho al esparcimiento con el convenio No52 sobre vacaciones anuales, el cual establecía que los trabajadores tenían derecho a vacaciones anuales pagadas de seis días al menos. Esta convención relanzó la discusión sobre el tema, por lo cual la OIT decidió crear en 1936 la Comisión Consultiva de Correspondencia para el Recreo de los Trabajadores (denominada luego Comisión de Recreos) responsable de estudiar "los problemas que implica la creación de servicios recreativos para los grandes grupos de población que habían adquirido el derecho de disfrutar unas vacaciones anuales pagadas y asimismo las recomendaciones sobre cuestiones tales como publicidad, viajes, alojamientos y recreos"62. La Sociedad de las Naciones en 1937 definió por primera vez al turista como aquel que viaja por placer, abandonando su lugar de residencia por más de veinte y cuatro horas, diferenciándolo del excursionista que no pernocta ${ }^{63}$.

Los principios del tiempo libre y el derecho al esparcimiento fueron consagrados en la Conferencia de Filadelfia de 1944, la que obliga a la OIT a fomentar programas que ofrezcan pasamientos y recreo a los trabajadores, así como en la Declaración Universal de los Derechos Humanos de 1948, la cual señala en su artículo 24, el derecho al descanso y el disfrute del tiempo libre.

\section{Conclusiones}

El balance historiográfico del presente artículo da cuenta de una importante cantidad de publicaciones en torno a las prácticas recreativas en el continente latinoamericano, aunque muchas de ellas se enmarcan en los enfoques socioculturales de la historia del movimiento obrero de vertiente más clásica. Es por ello que el estudio de las prácticas recreativas resulta una buena entrada para repensar la historia del trabajo, que supere, por una parte, una historia muy vinculada a la militancia obrera (tanto sindical como de partidos) y, por otra, a lo que ocurría al interior de la fábrica. Los historiadores se han interesado en investigar este campo por la multiplicidad de enfoques que permite, sintiéndose muy cómodos al analizar sus dimensiones políticas, sociales, culturales o económicas. En cierta forma, es probable que esta multiplicidad de abordajes y la multidimensión que adquieren las prácticas recreativas, explique, en parte, la fragmentación que se observa en los estudios que abordan la temática.

En este recuento historiográfico se ha puesto especial atención en la importancia del Estado y del empresariado en promover las prácticas recreativas, interesados en promover una política de promoción social y económica en torno al tiempo libre y el turismo. Las investigaciones que se han abocado a estudiar las prácticas recreativas por parte de los propios trabajadores, las inscriben dentro de ciertos marcos ideológicos de raigambre

61 OIT, El tiempo libre de los trabajadores. Una bibliografía con notas, Ginebra, OIT, 1967, p. 2.

62 Ibid.

63 Marc Boyer, Le Tourisme, Paris, Seuil, 1982. 
anarquista, socialista o comunista. Sin embargo, se hace necesario complejizar al sujeto trabajador, entendiéndolo no solo como sujeto de derechos laborales, sino también como sujeto consumidor. Los estudios enmarcados en los enfoques económicos permiten observar que las mejoras salariales y los programas de control de precios y de incentivo al consumo posibilitaron a los trabajadores el acceso a bienes materiales y culturales, así como el disfrute de mayor tiempo libre a partir de la década de 1930.

Sin desconocer los aportes de los estudios sociales y culturales enmarcados en la "historia heroica" del movimiento obrero, y en aquellos trabajos de microhistoria que han estudiado las fábricas y complejos industriales, el presente recuento historiográfico muestra la ausencia de perspectivas comparadas y de enfoques transnacionales al momento de abordar las prácticas recreativas. Claramente estos enfoques enriquecerían las historias nacionales y permitirían hacer comprensible una historia del trabajo a nivel latinoamericano, que piense al trabajo como una categoría histórica sustancial y no solo al trabajador, lo que supone articular al menos tres órdenes de problemas históricos: la extensión de los derechos sociales, la organización del trabajo y la producción, y el movimiento obrero.

\section{Bibliografía}

Archila, Mauricio (1991), “El uso del tiempo libre de los obreros. 1910-1945”, Anuario Colombiano de Historia Social y de la Cultura, Nº18-19, pp. 145-184.

Armas, Fernando (2018). Una historia del turismo en el Perú. El Estado, los visitantes y los empresarios (1800-2000) (Lima: USMP) Vol. 1-2.

Baranowski, Shelley (2004). Strength Through Joy: Consumerism and Mass Tourism in the Third Reich. Cambridge: Cambridge University Press.

Boyer, Marc (1982). Le Tourisme. Seuil : Paris.

Booth, Rodrigo (2014) "Turismo, panamericanismo e ingeniería civil: la construcción del camino escénico entre Viña del Mar y Concón (1917-1931)”. Historia N47, pp.277-311

Bravo Elizondo, Pedro, (1986), Cultura y teatro obreros en Chile, 1900-1930, Madrid: Ediciones Michay.

Camarero, Hernán, (2005), “Experiencias de cultura obrera: las prácticas de entretenimiento y erudición de los socialistas y los comunistas durante los años 19201930", X Jornadas Interescuelas/Departamentos de Historia. Escuela de Historia de la Facultad de Humanidades y Artes, Universidad Nacional del Rosario. Departamento de Historia de la Facultad de Ciencias de la Educación, Universidad Nacional del Litoral, Rosario.

Camarero, Hernán (2007), A la conquista de la clase obrera. Los comunistas y el mundo del trabajo en la Argentina, 1920-1935. Buenos Aires: Siglo XXI Editores.

Capanegra, César (2006), "La política turística en la Argentina en el siglo XX". Aportes y transferencias, Nº1, pp. 43-61.

Castel, Robert (1997), Las metamorfosis de la cuestión social. Una crónica del salariado. Buenos aires: Paidós.

Cicalese, G. (2002), “Conflictos políticos, enredos jurídicos y negocios de verano en torno de las playas marplatenses". En Elisa Pastoriza, Las puertas al mar. Consumo, ocio y 
política en Mar del Plata, Montevideo y Viña del Mar. Buenos Aires: Editorial Biblos, pp. 133162.

Comparato, Guillermo (2014), “Matices populistas: La política turística de Getulio Vargas (1937-1954) y de Juan Domingo Perón (1946-1952)". Trashumante - Revista Americana de Historial Social N³, pp. 16-133.

Corbin, Alain (1993), El territorio del vacío. Occidente y la invención de la playa. Barcelona: Mondadori Grijalbo.

Da Cunha, Nelly (2010), Montevideo ciudad balnearia (1900-1950). El municipio y el fomento del turismo. Montevideo: Facultad de Humanidades y Ciencias de la Educación.

Drinot, Paulo y Knight, Alan. (2015), La Gran Depresión en América Latina. México: FCE.

Elias, Norbert y Dunning, Eric (1992), Deporte y ocio en el proceso de la civilización. México: FCE.

Faraldo, José y Carolina Rodríguez-López (2013), Introducción a la historia del turismo. Madrid: Alianza Editorial.

Grazia, Victoria de (1981), The Culture of Consent: Mass Organization of Leisure in Fascist Italy. New York, Cambridge University Press.

Grez, Sergio, Los anarquistas y el movimiento obrero. La alborada de "la Idea" en Chile, 1893-1915, Santiago: Lom Ediciones, 2007.

Grez, Sergio (2011), “¿Teatro ácrata o teatro obrero? Chile, 1895-1927”, Estudios Avanzados, N¹5, pp. 9-29.

Giucci, G. (2007), La vida cultural del automóvil. Rutas de la modernidad cinética. Buenos Aires: Universidad Nacional de Quilmes-Prometeo.

Godoy, Eduardo (2008), “El discurso moral de los anarquistas chilenos en torno al alcohol a comienzos del siglo XX", en Juan Carlos Yáñez (Editor), Alcohol y trabajo. El alcohol y la formación de las identidades laborales en Chile. Siglo XIX y XX, Osorno: Universidad de Los Lagos, pp. 121-144.

Gordon, Bertram (2002), “El turismo de masas: un concepto problemático en la historia del siglo XX", Historia Contemporánea, N²5, pp. 125-156.

Hobsbawm, Eric (1987), El mundo del trabajo. Estudios históricos sobre la formación y evolución de la clase obrera. Madrid: Crítica.

Jones, Gareth Stedman (1989), Lenguajes de clase. Estudios sobre la historia de la clase obrera inglesa. Madrid: Siglo XXI.

Leonardi, Yanina (2014), “Ocio y arte para los obreros durante el primer peronismo (1946-1955)", Revista Mundos do Trabalho, N¹2, pp. 239-249.

Linden, M. (2006), Historia transnacional del trabajo. Valencia: ned-Instituto de Historia Social.

Lvovich, D. y J. Suriano (2006), Las políticas sociales en perspectiva histórica. Argentina, 1870-1952 Buenos Aires: Prometeo.

Milanesio, Natalia (2014), Cuando los trabajadores salieron de compras. Nuevos consumidores, publicidad y cambio cultural durante el primer peronismo. Buenos Aires: Siglo XXI Editores.

Núñez, Paula (2018), “Marcas y atractivos en una provincia imaginada. Río Negro, Argentina, 1958-1976", Quinto Sol, N¹, pp. 1-24. 
OIT (1936), “Convenio sobre las vacaciones pagadas". (N52), ILO: Ginebra

Osow, Silvina (2010), El desarrollo turístico en la Argentina durante el siglo XX: La política turística peronista (1946-1955). Tesis para obtener el grado de Licenciada en Sociología, Universidad Nacional de La Plata.

Ospital, Silvia (2005), “Turismo y territorio nacional en Argentina. Actores sociales y políticas públicas, 1920-1940". Estudios Interdisciplinarios de América Latina y el Caribe, N², pp. 63-84.

Pastoriza, Elisa (2002), Las puertas al mar. Consumo, ocio y politica en Mar del Plata, Montevideo y Viña del Mar, Buenos Aires: Editorial Biblos.

Pastoriza, Elisa (2008), "El turismo social en la Argentina durante el primer peronismo. Mar del Plata, la conquista de las vacaciones y los nuevos rituales obreros, 1943-1955". Revista Nuevo Mundo, Débats, publicada el 16 de junio de 2008. URL: http:/ / nuevomundo.revues.org/36472

Pastoriza, Elisa (2012), “Nuevos objetos de la Historia: los estudios turísticos en una perspectiva comparada", Anuario IEHS, N²7, pp. 323-330.

Pereira, Sergio, (2005), Antología crítica de la dramaturgia anarquista en Chile. Santiago: Editorial de la Universidad de Santiago.

Piglia, Melina (2008), “La incidencia del Touring Club y del ACA en la construcción del turismo como cuestión pública". Estudios y perspectiva en turismo, vol 17, pp. 51-70.

Piglia, Melina (2012), “En torno a los Parques Nacionales: primeras experiencias de una política turística nacional centralizada en la Argentina (1934-1950)", Pasos, N¹, pp. 6173.

Porrini, Rodolfo (2011), "Izquierda uruguaya y culturas obreras. Propuestas al 'aire libre': pic-nics y paseos campestres en Montevideo: 1920-1950". Revista Mundos do Trabalho 3(6): pp. 105-129.

Porrini, Rodolfo (2020), “Formas de la cultura alternativa: las 'veladas' y los festivales de las izquierdas uruguayas (Montevideo, 1920-1950), Izquierdas, N49, pp. 222-242.

Ramacciotti, Karina (2009), La política sanitaria del peronismo. Buenos Aires: Editorial Biblos.

Rinke, Stephan (2014), Encuentros con el yanqui: Norteamericanización y cambio sociocultural en Chile (1898-1990). Santiago: Dibam.

Rosemblatt, K. (1995), “Masculinidad y trabajo: el salario familiar y el estado de compromiso, 1930-1950". Proposiciones N²6, pp. 70-86

Scarzanella, E. (1988), “El ocio peronista: vacaciones y "turismo popular" en Argentina (1943-1955)". Entrepasados N¹4, pp. 65-84.

Schaffer, M. (2001), See America first: Tourism and national identity 1880-1940. Washington DC: Smithsonian Institution Press.

Sgrazzutti, Jorge Pedro (2004). "La organización del "Tiempo Libre" en dictaduras europeas y en Argentina entre 1922 y 1955”, Revista Historia Moderna i Contemporània, N², pp. 17-61.

Silva, Bárbara (2018), "La espacialidad y el paisaje en las representaciones nacionales durante el Frente Popular chileno, 1938-1941", Revista de Historia Social y de las Mentalidades, N¹, pp. 129-153. 
Silva, Bárbara y Rodrigo Henríquez (2017), “El pueblo del Frente. Representaciones sobre la ciudadanía en Chile: 1930-1950”, ERLACS, Nº103, pp. 91-108.

Silvestre, G. (1999) “Postales argentinas". En: Altamirano, C. (Ed.) La Argentina en el siglo XX. Buenos Aires: Ariel, pp. 111-135.

Simonassi, Silvia y Laura Badaloni (2013), “Trabajadores, empresas y comunidades urbanas: reflexiones introductorias", Avances del Cesor n¹0: 101-111.

Smith, M. (2003). Issues in cultural tourism studies. Nueva York: Routledge.

Thompson, E. P. (2012). La formación de la clase obrera en Inglaterra. Madrid: Capitán Swing.

Tizzoni, E. (2014) "Les politiques touristiques du fascisme et les relations internationales de l'Italie, entre diplomatie publique et création d'une marque de destination-Italie". Cahiers de la Méditerranée, N88, pp. 1-15

Torre, J. C. y Pastoriza, E. (2002) “La democratización del bienestar". En: Torre, J. C. (Dir.) Los años peronistas (1943-1955). Buenos Aires: Editorial Sudamericana, pp. 257-312

Unión Panamericana (1933). Séptima Conferencia Internacional Americana. Montevideo: OIT.

Urry, J. (1996) O olhar do turista: lazer e viagens nas sociedades contemporâneas. São Paulo: SESC/ Nobel.

Vejsbjerg, L., Núñez, P. y Matossian, B. (2014) “Transformation of Frontier National Parks into tourism sites. The North Andean Patagonia Experience (1934-1955)". Alma Tourism, $\mathrm{N}^{\circ} 10$, pp. 1-22.

Videla, Enzo, Hernán Venegas y Milton Godoy (edit.), (2016), El orden fabril. Paternalismo industrial en la minería chilena. 1900-1950. Valparaíso: Editorial América en movimiento.

Walton, John y James Walvin (edit.) (1983). Leisure in Britain, 1788-1939. Manchester: Manchester University Press, Oxford Road.

Walton, John (2000), The British Seaside: holidays and resorts in the twentieth century. Manchester: Manchester University Press.

Yáñez, Juan Carlos (2015) “El control del tiempo de trabajo. El tiempo de trabajo. El establecimiento de la jornada laboral continua en Chile en 1942". Revista de Derecho, XLV: pp. 403-422.

Yáñez, Juan Carlos (2016) “Trabajo y políticas culturales sobre el tiempo libre. Santiago de Chile. Década de 1930", Historia 49(2): pp. 595-629.

Yáñez, Juan Carlos (2017) “El Instituto de Educación Hotelera de Chile: Una experiencia pionera de formación en el campo de la hospitalidad". Estudios y Perspectivas en Turismo, №1, pp. 178-193.

Zuelow, E. (2016) A history of modern tourism. New York: Palgrave. 